\title{
Discussion of 011405JOR by Colombo and Del Gado
}

Citation: Journal of Rheology 58, 1117 (2014); doi: 10.1122/1.4892862

View online: https://doi.org/10.1122/1.4892862

View Table of Contents: http://sor.scitation.org/toc/jor/58/5

Published by the The Society of Rheology

\section{Articles you may be interested in}

Direct observation of DNA dynamics in semidilute solutions in extensional flow Journal of Rheology 61, 151 (2017); 10.1122/1.4972236

Parameter-free prediction of DNA dynamics in planar extensional flow of semidilute solutions

Journal of Rheology 61, 169 (2017); 10.1122/1.4972237

The performance of the hot end in a plasticating 3D printer Journal of Rheology 61, 229 (2017); 10.1122/1.4973852

Thermokinematic memory and the thixotropic elasto-viscoplasticity of waxy crude oils Journal of Rheology 61, 427 (2017); 10.1122/1.4978259

Discussion of 019405JOR by R. Zia et al.

Journal of Rheology 58, 1158 (2014); 10.1122/1.4892821

Viscoelasticity of entangled random polystyrene ionomers Journal of Rheology 60, 1031 (2016); 10.1122/1.4955432

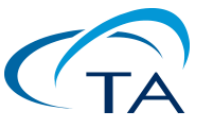

The WORLD'S most

VERSATILE platform for RHEOLOGICAL MEASUREMENTS

The Discovery Hybrid Rheometer
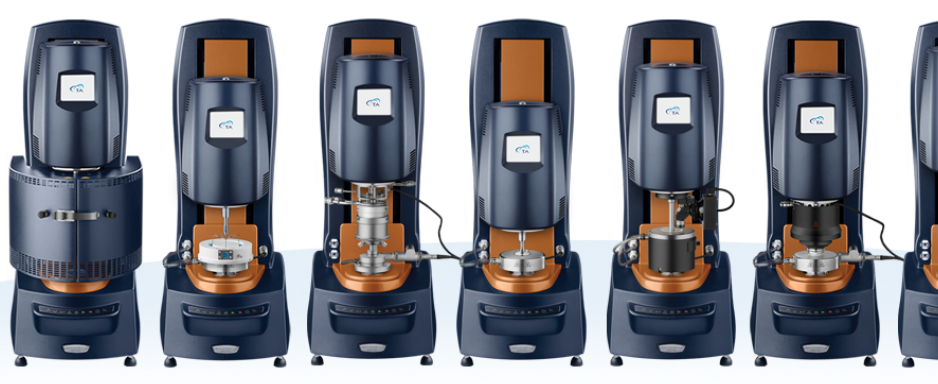


\section{Discussion of 011405JOR by Colombo and Del Gado}

Question: Alessio Zaccone: Is it possible to formulate a simple one-on-one correspondence between structural/mechanical heterogeneity, and the shear-banding phenomenon? In other words, is there strong correlation between the spatial distribution of less dense, highly nonaffine regions and the regions where shear-bands appear? Or is the balance more subtle and requires a more complex coupling?

Author Response: Colombo and Del Gado: There seems to be indeed a direct correlation between the velocity profiles indicating the flow inhomogeneity and the appearance of a region from which particles (and bonds) are depleted (see Fig. 11 in the manuscript). With respect to nonaffine displacements, we have not characterized their spatial distribution. We could tentatively speculate that they should increase in the particle depleted region, whereas it could be that the more densely connected regions could be characterized by a relatively more affine response, but this needs to be verified.

Question: Roger Bonnecaze: From your understanding of the interplay among interparticle forces, microstructural dynamics, and macroscopic properties of attractive colloidal gels, how would you advise users to formulate the gels to achieve desired properties such as moduli, yield stress, yield strain, and viscosity? In short, how does microscale understanding inform design of these important materials?

Author Response: Colombo and Del Gado: There is still much to be done to get to a quantitative knowledge and to translate it into practical indications, but we are on a good track. There is a great potential, especially in combining numerical simulations with experiments. Theoretical and simulations predictions on how the microstructure can affect the linear response of these materials have already been developed. The results we have discussed in the contribution to this Special Issue, moreover, indicate that common assumptions used to interpret the rheological behavior should be rethought of, for example, when inferring a rather homogeneous fluidification of the microstructure under shear. They also give a new microscopic insight into how complex reorganization processes during yielding might lead to the development of flow instabilities.

Question: George Petekidis: Do you think that hydrodynamic interactions would affect not only the microscopic dynamics and time evolution of the gel coarsening (ageing) but also the structure of the metastable gel state at rest or under shear? Computer simulations by Furukawa et al. (2010) suggest that the latter might be possible.

Author Response: Colombo and Del Gado: Hydrodynamic interactions most probably affect the intermediate stages of aggregation during the gel formation, as in fact suggested by the work you mention. For our model gel, we do not expect them to qualitatively change the final structure of the gel network at rest, since we do not see any significant changes in simulations performed integrating different equations of motion (from Newtonian to Langevin dynamics) and varying the simulation time window of 
several order of magnitude. Nevertheless, this expectation remains to be verified. Concerning the gel under shear, again hydrodynamic interactions are certainly likely to play a role, although they might not qualitatively change the very long shear rate regime, because there the gel restructuring seems to be dominated by elastic correlations. Hydrodynamic interactions, on the other hand, might well influence the structure of the gel under shear upon increasing shear rate. Properly including hydrodynamics in the type of simulations we have carried out is quite challenging, but we would be very interested in exploring possible solutions.

Question: Dimitris Vlassopoulos: The work of Zia et al. (019405JOR) suggests the presence of one dominant length-scale in the gel. Your work suggest a gradual yielding process, implying multiple length scales. How can we reconcile this? In other words, what is the reason for one length scale in a gel (irrespectively of coarsening or differences in volume fraction/strength of attraction). Or is it system specific?

Author Response: Colombo and Del Gado: It would be worth trying to further investigate dynamical correlations in the aging gel in Zia's work, because they might reveal a more complex scenario (e.g., longer range correlations or again multiple length scales) than the one emerging from the analysis of the contribution to the overall mean square displacement from populations of particles with different coordination number. Suggestions in this direction can be found in our recent works [Colombo et al. (2013); Colombo and Del Gado (2014)]. Aside from this, it could also be that a qualitative different picture holds depending on the strength of the attractive interactions (rather than being system specific): Deeply quenched gels might be characterized by a more complex microscopic dynamics and structure/dynamics interplay with respect to rather weakly attractive gels (i.e., where the attraction strength is not larger than $2-3 k_{B} T$, as probably considered in Zia's work).

Question: Marco Laurati: In the simulated system, the authors assumed that Brownian-motion induced rearrangements are negligible due to the strong binding energy between particles. I wonder if for strains larger than the yield strain of the system, i.e., when the network structure starts to rupture, this assumption will still hold or if breaking of interparticle bonds would lead to a relevant contribution of Brownian motion in experimental systems in this range of strains. What could be the effect (if any) on the obtained results?

Author Response: Colombo and Del Gado: After the yielding point, when the gel is flowing, the presence of a finite temperature is certainly expected to play a role. The importance of Brownian motion should depend on the ratio between the shear rate and the rate of the thermally induced breakage of the gel bonds. One can get an idea of the restructuring processes induced by thermal fluctuations from our work on the same model gel at finite temperature at rest. We are currently investigating the gel at finite temperature under shear.

Question: Ralph Colby: Your simulations suggest a very complicated and rich behavior of colloidal gels that the simple models available now cannot possibly capture. To what extent do your results and typical experimental results depend on the starting state of the gel? Does $k_{\mathrm{B}} T / \varepsilon=0.05$ allow your simulation to fully equilibrate and satisfy detailed balance? How large does $k_{\mathrm{B}} T / \varepsilon$ need to be for experiments to equilibrate, given their longer waiting time? 
Author Response: Colombo and Del Gado: Because the gel corresponds to a metastable state, we expect the results to depend on its starting state. This feature should be inherent in the physics of the system. Hence the choice $k_{\mathrm{B}} T / \varepsilon=0.05$ does not allow for true, full equilibration. Nevertheless, in order to control the influence of the initial state and have reproducible results, we have obtained the initial state of the gel via a very slow cooling (as opposed to a fast quench) at the end of which we do not detect any significant aging of the gel within the accessible simulation time window, and the gel structure does not show any appreciable change upon changing the cooling rate within the numerically accessible range. We expect this situation to be relevant to experimental conditions, where the typical observation time scale is much larger than the one needed for the gel to form and the gel is usually considered as stable. Starting from the results obtained in these conditions, we plan to investigate how deeper quenches and less stable (or steadystate) gel structures may lead to different scenarios.

\section{References}

Furukawa, A., and H. Tanaka, "Key role of hydrodynamic interactions in colloidal gelation," Phys. Rev. Lett. 104, 245702 (2010).

Colombo, J., and E. Del Gado, "Self-assembly and cooperative dynamics of a model colloidal gel network," Soft Matter 10(22), 4003-4015 (2014).

Colombo, J., A. Widmer-Cooper, and E. Del Gado, "Microscopic picture of cooperative processes in restructuring gel networks," Phys. Rev. Lett. 110, 198301 (2013). 